\title{
ON FACTORIZATION OF $p$-ADIC MEROMORPHIC FUNCTIONS
}

\author{
B. SAOUDI, A. BOUTABAA and T. ZERZAIHI
}

February 6, 2019

\begin{abstract}
In this paper, we study primeness and pseudo primeness of $p$-adic meromorphic functions. We also consider left (resp. right) primeness of these functions. We give, in particular, sufficient conditions for a meromorphic function to satisfy such properties. Finally, we consider the problem of permutability of entire functions.
\end{abstract}

\section{Introduction}

For every prime number $p$, we denote by $\mathbb{Q}_{p}$ the field of $p$-adic numbers, and we denote by $\mathbb{C}_{p}$ the completion of an algebraic closure of $\mathbb{Q}_{p}$, which is endowed with the usual $p$-adic absolute value. Given $a \in \mathbb{C}_{p}$ and $r>0, d(a, r)$ and $d\left(a, r^{-}\right)$are respectively the disks $\left\{x \in \mathbb{C}_{p} /|x-a| \leq r\right\}$ and $\left\{x \in \mathbb{C}_{p} /|x-a|<r\right\}$; and $C(a, r)$ is the circle $\left\{x \in \mathbb{C}_{p} /|x-a|=r\right\}=d(a, r) \backslash d\left(a, r^{-}\right)$. It is easily seen that, two disks have a non-empty intersection if and only if they are nested (i.e, one of them is contained in the other).

We denote by $\mathcal{A}\left(\mathbb{C}_{p}\right)$ the $\mathbb{C}_{p}$-algebra of entire functions in $\mathbb{C}_{p}$ and $\mathcal{M}\left(\mathbb{C}_{p}\right)$ the field of meromorphic functions in $\mathbb{C}_{p}$, i.e, the field of fractions of $\mathcal{A}\left(\mathbb{C}_{p}\right)$. Let $f(x)=\sum_{n \geq 0} a_{n} x^{n}$ be a $p$-adic entire function. For all $r>0$, we denote by

Mathematics Subject Classification (2010): Primary 30D05, Secondary 30D35.

keywords: p-adic meromorphic function, primeness, right-primeness, left-primeness 
$|f|(r)=\max _{n \geq 0}\left|a_{n}\right| r^{n}$, the maximum modulus of $f$. This is extended to meromorphic functions $h=f / g$ by $|h|(r)=|f|(r) /|g|(r)$.

An element $f \in \mathcal{A}\left(\mathbb{C}_{p}\right)$ (resp. $f \in \mathcal{M}\left(\mathbb{C}_{p}\right)$ ) is said to be transcendental if it is not a polynomial (resp. rational function). Thus, a $p$-adic transcendental meromorphic function admits infinitely many zeros or infinitely many poles or both. It should also be noted that a transcendental entire function $f$ has no exceptional value or Picard value in $\mathbb{C}_{p}$, so for every $\beta \in \mathbb{C}_{p}$, the function $f-\beta$ has infinitely many zeros.

Recall also that, if $D$ is a disk and $f$ an analytic function in $D$, then $f(D)$ is a disk. Moreover if $f^{\prime}$ has no zero in $D$, there exist a disk $d \subset D$ such that the restiction $f_{\mid d}: d \longrightarrow f(d)$ is bi-analytic. This means that $f_{\mid d}$ is an analytic bijection and that its reciprocal function $f_{\mid d}^{-1}$ is also analytic [4].

Let $F, f, g$ be $p$-adic meromorphic functions such that $F=f \circ g$. We say that $f$ and $g$ are respectively left and right factors of $F$. The function $F$ is said to be prime (resp. pseudo-prime, resp. left-prime, resp. right-prime) if every factorization of $F$ of the above form implies that either $f$ or $g$ is a linear rational function (resp. $f$ or $g$ is a rational function, resp. $f$ is a linear rational function whenever $g$ is transcendental, $g$ is linear whenever $f$ is transcendental). If the factors are restricted to entire functions, the factorization is said to be in the entire sense. The article by Bézivin and Boutabaa [2] is to our knowledge the only work dedicated to the study of factorization of $p$-adic meromorphic functions. They show, in particular, that if an entire function $F$ is prime (resp pseudo-prime) in $\mathcal{A}\left(\mathbb{C}_{p}\right)$, then $F$ is prime (resp pseudo-prime) in $\mathcal{M}\left(\mathbb{C}_{p}\right)$. This is false in the field $\mathbb{C}$ of complex numbers. Indeed, Ozawa in [6] gives examples of complex entire functions that are prime in $\mathcal{A}(\mathbb{C})$ without being prime in $\mathcal{M}(\mathbb{C})$.

In this article, we provide sufficient conditions for a $p$-adic meromorphic function to be prime or pseudo-prime. We give examples of meromorphic functions satisfying these conditions.

We also show that almost all $p$-adic transcendental entire functions are prime, in the sense that it is most often enough to add or multiply these functions by an affinity to obtain a prime entire function.

Finally, we briefly discuss the question of permutability of entire functions. Or, 
in other words, one wonders: when do we have $f \circ g=g \circ f$ for $p$-adic entire functions $f$ and $g$ ?

Our method is based on the distribution of zeros and poles of the considered functions as well as the properties of their maximum modulus.

\section{PRIMALITY AND PSEUDO PRIMALITY}

Let us first prove the following result which gives sufficient conditions for a $p$-adic meromorphic function to be pseudo-prime.

Theorem 2.1. Let $F$ be a transcendental meromorphic function in $\mathbb{C}_{p}$ whose all poles are simple except a finite number of them. Suppose moreover that, for every $\beta \in \mathbb{C}_{p}$, all the zeros of the function $F-\beta$ are simple except a finite number of them. Then $F$ is pseudo-prime.

To prove Theorem 2.1 we need the following lemma, whose proof is given in [5]

Lemma 2.2. Let $f_{1}, f_{2} \in \mathcal{A}\left(\mathbb{C}_{p}\right)$ such that $f_{1}^{\prime} f_{2}-f_{1} f_{2}^{\prime} \equiv c ; \quad c \in \mathbb{C}_{p}$. So, if one of the functions $f_{1}, f_{2}$ is not affine then $c=0$ and $f_{1} / f_{2}$ is a constant.

We also need the following lemma, whose proof is given in [2]

Lemma 2.3. Let $F, f, g$ be three meromorphic functions in $\mathbb{C}_{p}$. Suppose that $f$ is not a rational function and that $F=f \circ g$. Then $g$ entire.

Proof of Theorem 2.1.

Suppose that $F$ is not pseudo-prime. Hence there exist two transcendental meromorphic functions $f$ and $g$ such that $F=f \circ g$. Then, by Lemma 2.3, the function $g$ is entire. Let us write $f$ in the form $f=f_{1} / f_{2}$ where $f_{1}, f_{2}$ are entire functions with no common zeros. As $f$ is transcendental, we see that at least one of the functions $f_{1}, f_{2}$ is transcendental. So by Lemma $2.2, f_{1}^{\prime} f_{2}-f_{1} f_{2}^{\prime}$ is a non-constant entire function, hence admits at least a zero $\alpha$. Let us distinguish the two following cases:

1. If $f_{2}(\alpha)=0$, then $f_{1}(\alpha) \neq 0$ and $f_{2}^{\prime}(\alpha)=0$, so $\alpha$ is a multiple zero of $f_{2}$ and all element of the set $g^{-1}(\{\alpha\})$ are multiple zeros of $f_{2} \circ g$ and are multiple poles of 
$F$. Since $g \in \mathcal{A}\left(\mathbb{C}_{p}\right) \backslash \mathbb{C}_{p}[X]$, then the set $g^{-1}(\{\alpha\})$ is infinite and $F$ has infinitely many multiple poles, which is a contradiction.

2. If $f_{2}(\alpha) \neq 0$, then $f^{\prime}(\alpha)=\left(f_{1}^{\prime} f_{2}-f_{1} f_{2}^{\prime}\right)(\alpha) / f_{2}(\alpha)=0$. Let $\beta=f(\alpha)$, then $\alpha$ is a multiple zero of $f-\beta$. But the equation $g(x)=\alpha$ admits infinitely many solutions and for every such solution $\omega$ we have:

$$
\left\{\begin{array}{l}
(F-\beta)(\omega)=F(\omega)-\beta=f \circ g(\omega)-\beta=f(\alpha)-\beta=0, \\
(F-\beta)^{\prime}(\omega)=F^{\prime}(\omega)=f^{\prime}(g(\omega)) \times g^{\prime}(\omega)=f^{\prime}(\alpha) \times g^{\prime}(\omega)=0 .
\end{array}\right.
$$

Then $F-\beta$ has a infinitely many zeros, which is a contradiction again.

Hence $F$ is pseudo-prime.

Theorem 2.1. provides, in particular, necessary conditions for the pseudo-primeness of $p$-adic entire functions, which are summarized in the following corollary:

Corollary 2.4. Let $F \in \mathcal{A}\left(\mathbb{C}_{p}\right) \backslash \mathbb{C}_{p}[X]$ be such that, for every $\beta \in \mathbb{C}_{p}$, all the zeros of the function $F-\beta$ are simple except a finite number of them. Then $F$ is pseudo-prime.

The following results give more information about the left factor in any factorization of a $p$-adic entire function that satisfies the above conditions.

Theorem 2.5. Let $F \in \mathcal{A}\left(\mathbb{C}_{p}\right) \backslash \mathbb{C}_{p}[X]$ be such that for every $\beta \in \mathbb{C}_{p}$, only finitely many zeros of $F-\beta$ are multiple. Then $F$ is left-prime.

Proof. Suppose that $F=f \circ g$ where $g \in \mathcal{A}\left(\mathbb{C}_{p}\right) \backslash \mathbb{C}_{p}[X]$ and $f \in \mathcal{A}\left(\mathbb{C}_{p}\right)$. By Corollary 2.4, we know that $F$ is pseudo-prime. So $f$ is a polynomial. Suppose that $\operatorname{deg} f \geq 2$, then $f^{\prime}$ has at least one zero $\alpha$. Since $g \in \mathcal{A}\left(\mathbb{C}_{p}\right) \backslash \mathbb{C}_{p}[X]$, the set $W=\left\{x \in \mathbb{C}_{p} / g(x)=\alpha\right\}$ is infinite.

Let $\beta=f(\alpha)$. Then, for every $\omega \in W$, we have:

$$
\left\{\begin{array}{l}
((F-\beta)(\omega)=F(\omega)-\beta=f \circ g(\omega)-\beta=f(\alpha)-\beta=0, \\
(F-\beta)^{\prime}(\omega)=F^{\prime}(\omega)=f^{\prime}(g(\omega)) \times g^{\prime}(\omega)=f^{\prime}(\alpha) \times g^{\prime}(\omega)=0 .
\end{array}\right.
$$

This means that $F-\beta$ has infinitely many multiple zeros, a contradiction. Hence the left-primeness of $f$ is proven.

Theorem 2.6. Let $F \in \mathcal{A}\left(\mathbb{C}_{p}\right) \backslash \mathbb{C}_{p}[X]$ be such that for every $\beta \in \mathbb{C}_{p}$, the function $F-\beta$ has at most one multiple zero. Then $F$ is prime. 
Proof. By Theorem 2.5, we already see that $F$ is left-prime. So, it remains to show the right-primeness of $F$. For that, suppose that $F(z)=f \circ g$, where $f \in$ $\mathcal{A}\left(\mathbb{C}_{p}\right) \backslash \mathbb{C}_{p}[X]$ and $g \in \mathcal{A}\left(\mathbb{C}_{p}\right)$. From Corollary 2.4, we know that $F$ is pseudoprime. So $g$ is a polynomial. Suppose that $\operatorname{deg} g=d \geq 2$. We have $F^{\prime}(z)=$ $f^{\prime}(g(z)) g^{\prime}(z)$. Since $f \in \mathcal{A}\left(\mathbb{C}_{p}\right) \backslash \mathbb{C}_{p}[X]$, the function $f^{\prime}$ has infinitely many zeros. So we may choose an element $w \in \mathbb{C}_{p}$ such that $f^{\prime}(w)=0$ and $g-w$ has only simple zeros $\gamma_{1}, \ldots, \gamma_{d}$. Then for $i=1, \ldots, d$, we have $\left\{\begin{array}{l}F\left(\gamma_{i}\right)=f(w)=\beta \\ (F-\beta)^{\prime}\left(\gamma_{i}\right)=0\end{array}\right.$, which means $\gamma_{1}, \ldots, \gamma_{d}$ are multiple zeros of $F-\beta$, a contradiction. Hence $F(z)$ is right-prime.

Theorem 2.7. Let $F$ be a p-adic transcendental meromorphic function that admits at most finitely many poles. Suppose that for every $\beta \in \mathbb{C}_{p}$, only finitely many zeros of $F-\beta$ are multiple. Then $F$ is right-prime.

To prove this theorem, we need the following lemma whose proof is given in Bézivin [3]. It is more general than Lemma 2.2.

Lemma 2.8. Let $n \geq 1$ and let $f_{1}, \ldots, f_{n}$ be $p$-adic entire functions such that the wronskian $W\left(f_{1}, \ldots, f_{n}\right)$ is a non-zero polynomial. Then $f_{1}, \ldots, f_{n}$ are polynomials.

Proof of Theorem 2.7.

Suppose that $F(z)=f \circ g$, where $f \in \mathcal{M}\left(\mathbb{C}_{p}\right) \backslash \mathbb{C}_{p}(X)$ and $g \in \mathcal{M}\left(\mathbb{C}_{p}\right)$. By Theorem 2.1 the function $F$ is pseudo-prime. Then $g$ is a polynomial function. Suppose that $\operatorname{deg} g \geq 2$. Let us write $f$ in the form $f=f_{1} / f_{2}$ where $f_{1}, f_{2}$ are entire functions with no common zeros. We have $\left.f^{\prime}(z)=W\left(f_{2}, f_{1}\right) / f_{2}^{2}\right)(g(z)) g^{\prime}(z)$. As $f$ is transcendental and has a finite number of poles, we see that $f_{1}$ is transcendental and $f_{2}$ is polynomial. As $f \in \mathcal{M}\left(\mathbb{C}_{p}\right) \backslash \mathbb{C}_{p}(X)$, it follows by Lemma 2.8 that $W\left(f_{2}, f_{1}\right) \in \mathcal{A}\left(\mathbb{C}_{p}\right) \backslash \mathbb{C}_{p}(X)$ and admits then infinitely many zeros $\left\{w_{n}\right\}$ ( which are not zeros of $f_{2}$. For every integer $n$, big enough, the equation $g(z)=w_{n}$ admits at least two distinct roots, which are also common roots of $\left\{\begin{array}{l}F(z)=f\left(w_{n}\right) \\ F^{\prime}(z)=0\end{array}\right.$. Then we have a contradiction and $F(z)$ is right-prime. 
Theorem 2.9. Let $f(x)=\sum_{n \geq N} a_{n} x^{n}$ be a $p$-adic entire function such that $a_{n} \neq$ 0 , for every $n \geq N$. Suppose that there exists an integer $n_{0} \geq N$ such that $\left(\left|a_{n} / a_{n+1}\right|\right)_{n \geq n_{0}}$ is a strictly increasing unbounded sequence. Then the function $f$ is pseudo-prime.

To prove Theorem 2.9 we need the following lemma whose proof is given in [4].

Lemma 2.10. Let $f$ be a $p$-adic entire function defined by $f(x)=\sum_{n \geq N} a_{n} x^{n}$. Let $\mu$ and $\nu$ be, respectively, the smallest integer and the largest one such that $|f|(r)=$ $\left|a_{\mu}\right| r^{\mu}=\left|a_{\nu}\right| r^{\nu}$. Then:

i) $\mu$ is the number of zeros of $f$ in the disk $d\left(0, r^{-}\right)$;

ii) $\nu$ is the number of zeros of $f$ in the disk $d(0, r)$;

iii) $\nu-\mu$ is the number of zeros of $f$ in the circle $\mathcal{C}(0, r)$.

Proof of Theorem 2.9.

Let us, first set $r_{n}=\left|a_{n} / a_{n+1}\right|, \forall n \geq 0$. Quit to replace $n_{0}$ by a greater integer, we may assume that $n_{0}$ is such that: $r_{n_{0}}>\max \left\{r_{0}, \ldots, r_{n_{0}-1}\right\}$. In the sequel, we will show that $f$ has only simple zeros in $\mathbb{C}_{p} \backslash d\left(0, r_{n_{0}}\right)$. Indeed, let $r>r_{n_{0}}$. We distinguish two cases:

i) $r=r_{n}$ for some $n \geq n_{0}+1$. As the sequence $\left(r_{k}\right)_{k \geq n_{0}}$ is strictly increasing, we have $r_{n-1}<r_{n}<r_{n+1}$. We then have:

$$
\left|a_{n+1}\right| r_{n}^{n+1} /\left|a_{n}\right| r_{n}^{n}=\left|a_{n+1} / a_{n}\right| r_{n}=1 \text {, which means that }\left|a_{n}\right| r_{n}^{n}=\left|a_{n+1}\right| r_{n}^{n+1} \text {. }
$$

Moreover for every integer $\ell, 0 \leq \ell \leq n-1$, we have:

$$
\left|a_{\ell}\right| r_{n}^{\ell} /\left|a_{n}\right| r_{n}^{n}=\left|a_{\ell} / a_{n}\right| 1 / r_{n}^{n-\ell}=\left|a_{\ell} / a_{\ell+1}\right| \ldots\left|a_{n-1} / a_{n}\right| 1 / r_{n}^{n-k}<1 .
$$

Finally, for every integer $\ell, \ell>n+1$, we have:

$$
\begin{aligned}
\left|a_{\ell}\right| r_{n}^{\ell} /\left|a_{n+1}\right| r_{n}^{n+1}= & \left|a_{\ell} / a_{n+1}\right| r_{n}^{\ell-n-1} \\
& <\left|a_{\ell} / a_{n+1}\right|\left|a_{n+1} / a_{n+2}\right| \ldots\left|a_{\ell-1} a_{\ell}\right|=1 .
\end{aligned}
$$

Hence $|f|\left(r_{n}\right)=\max _{\ell \geq 0}\left|a_{\ell}\right| r_{n}^{\ell}$ is reached for the two values $\ell=n$ and $\ell=n+1$. This implies, by Lemm 2.10., that $f$ has only one zero in the circle $C\left(0, r_{n}\right)$.

ii) Suppose now that $r$ is different from $r_{n}$ for all $n>n_{0}$. Let $n \geq n_{0}$ be the sole integer such that $r_{n}<r<r_{n+1}$. We then have, for every integer $\ell, 0 \leq \ell \leq n$ :

$\left|a_{\ell}\right| r^{\ell}=\left|\frac{a_{\ell}}{a_{\ell+1}}\right|\left|\frac{a_{\ell+1}}{a_{\ell+2}}\right| \ldots\left|\frac{a_{n}}{a_{n+1}}\right|\left|a_{n+1}\right| r^{\ell} \leq\left|a_{n+1}\right| r_{n}^{n+1-\ell} r^{\ell}<\left|a_{n+1}\right| r^{n+1}$. 
Moreover for every integer $\ell>n+1$, we have:

$\frac{\left|a_{\ell}\right| r^{\ell}}{\left|a_{n+1}\right| r^{n+1}}=\frac{\left|a_{\ell}\right|}{\left|a_{n+1}\right|} r^{\ell-n-1}<\frac{\left|a_{\ell}\right|}{\left|a_{n+1}\right|} r_{n+1}^{\ell-n-1} \leq\left|\frac{a_{\ell}}{a_{n+1}}\right|\left|\frac{a_{n+1}}{a_{n+2}}\right| \ldots\left|\frac{a_{\ell-1}}{a_{\ell}}\right|=1$.

Then $|f|(r)=\max _{\ell \geq 0}\left|a_{\ell}\right| r^{\ell}$ is reached only for $\ell=n+1$. This implies, by Lemma 2.10, that $f$ has no zero in the circle $C(0, r)$. It follow that all the zeros of $f$ in $\mathbb{C}_{p} \backslash d\left(0, r_{n_{0}}\right)$ are simple. Now, for every $\beta \in \mathbb{C}_{p}$, there exists $r_{\beta}>0$ such that: $|f-\beta|(r)=|f|(r), \forall r>\max \left(r_{n_{0}}, r_{\beta}\right)$. It follows that $f-\beta$ has only simple zeros in $\mathbb{C}_{p} \backslash d\left(0, \max \left(r_{n_{0}}, r_{\beta}\right)\right)$. This means that all the possible multiple zeros of $f-\beta$ lie in $d\left(0, \max \left(r_{n_{0}}, r_{\beta}\right)\right)$ and are therefore finitely many. Using Corollary 2.4, we complete the proof of Theorem 2.9.

Corollary 2.11. Let $f$ be a p-adic entire function satisfying the conditions of Theorem 2.9., then for any non-zero polynomial $P$, the p-adic meromorphic function $g=f / P$ is pseudo-prime.

Proof. Note first that we may suppose that $f$ and $P$ have no common zeros. It is clear that $g$ has finitely many poles. Now let $\beta \in \mathbb{C}_{p}$. We see that the zeros of $g-\beta$ are the same as those of $f-\beta P$. Moreover, there exists $r_{\beta}>0$ such that for every $r>r_{\beta}$ we have: $|f-\beta P|(r)=|f|(r)$. It follows, by Theorem 2.9, that the function $f-\beta P$ (and therefore also $g-\beta$ ) has at most a finite number of multiple zeros. Thus by Theorem 2.1. the meromorphic function $g$ is pseudo-prime.

Corollary 2.12. Let $f(x)=\sum_{n \geq N} a_{n} x^{n}$ and $g(x)=\sum_{n \geq N} b_{n} x^{n}$ be two p-adic entire functions such that $a_{n} b_{n} \neq 0, \forall n \geq N$. Let $n_{0} \geq N$ be an integer such that the sequences $\left(\left|a_{n} / a_{n+1}\right|\right)_{n \geq n_{0}}$ and $\left(\left|b_{n} / b_{n+1}\right|\right)_{n \geq n_{0}}$ are strictly increasing and unbounded. Suppose moreover that $\lim _{r \rightarrow+\infty}(|f|(r) /|g|(r))=+\infty$. Then the meromorphic function $h=f / g$ is pseudo-prime.

Proof. Note first that we may suppose that the entire functions $f$ and $g$ have no common zeros. By Theorem 2.9, we see that the entire functions $f$ and $g$ have at most finitely many multiple zeros. Hence the meromorphic function $h=f / g$ has at most finitely many multiple zeros and poles.

Now let $\beta \in \mathbb{C}_{p}$. We see that the zeros of $h-\beta$ are the same as those of $f-\beta g$. Moreover, as $\lim _{r \rightarrow+\infty}(|f|(r) /|g|(r))=+\infty$, there exists $r_{\beta}>0$ such that for 
every $r>r_{\beta}$ we have: $|f-\beta g|(r)=|f|(r)$. It follows, by Theorem 2.9 , that the function $f-\beta g$ (and therefore also $h-\beta$ ) has at most a finite number of multiple zeros. Thus, by Theorem 2.1, the meromorphic function $h$ is pseudo-prime.

In what follows, we will provide examples of meromorphic $\mathrm{p}$-adic functions satisfying the conditions of Corollary 2.12. Let's first recall that, given a real number $x$, we call integer part of $x$ and we denote by $E(x)$ the unique integer such that $E(x) \leq x<E(x)+1$. It is easily shown that:

Lemma 2.13. For all real numbers $x$ and $y$, we have:

$$
E(x-y) \leq E(x)-E(y) \leq E(x-y)+1 .
$$

Proposition 2.14. Let $N$ be an integer $\geq 3$ and let $\alpha, \beta \in \mathbb{C}_{p}$ be such that $|\beta|<$ $|\alpha|<1$. Let $f$ and $g$ the functions defined by $f(x)=\sum_{n \geq 0} \alpha^{E\left((n / N)^{N}\right)} x^{n}$ and $g(x)=\sum_{n \geq 0} \beta^{E\left((n / N)^{N}\right)} x^{n}$. The meromorphic function $h=f / g$ is a pseudo-prime.

Proof. We have $f(x)=\sum_{n \geq 0} a_{n} x^{n}$, where $a_{n}=\alpha^{E\left((n / N)^{N}\right)}$ for every $n \geq 0$.

We easily check that, $\lim _{n \rightarrow+\infty}\left|a_{n}\right| r^{n}=\lim _{n \rightarrow+\infty}\left|\alpha^{E\left((n / N)^{N}\right)}\right| r^{n}=0$, for every $r>0$; which means that $f$ is an entire function in $\mathbb{C}_{p}$.

Let us now show that, if $n_{0}$ is an integer $\geq\left(2 N^{N-1} /(N-1)\right)^{1 /(N-2)}$, the sequence $\left(\left|a_{n} / a_{n+1}\right|\right)_{n \geq n_{0}}$ is strictly increasing.

For every $n \geq 0$, we have: $\left|a_{n} / a_{n+1}\right|=(1 /|\alpha|)^{E\left((n+1 / N)^{N}\right)-E\left((n / N)^{N}\right)}$.

As the real function $x \mapsto(1 /|\alpha|)^{x}$ is strictly increasing, it follows by Lemma 2.10. that:

$$
\left(\frac{1}{|\alpha|}\right)^{E\left(\left(\frac{n+1}{N}\right)^{N}-\left(\frac{n}{N}\right)^{N}\right)} \leq\left|\frac{a_{n}}{a_{n+1}}\right| \leq\left(\frac{1}{|\alpha|}\right)^{E\left(\left(\frac{n+1}{N}\right)^{N}-\left(\frac{n}{N}\right)^{N}\right)+1}
$$

In the same way, we have:

$$
\left(\frac{1}{|\alpha|}\right)^{E\left(\left(\frac{n+2}{N}\right)^{N}-\left(\frac{n+1}{N}\right)^{N}\right)} \leq\left|\frac{a_{n}+1}{a_{n+2}}\right| \leq\left(\frac{1}{|\alpha|}\right)^{E\left(\left(\frac{n+2}{N}\right)^{N}-\left(\frac{n+1}{N}\right)^{N}\right)+1}
$$

It follows from these last two inequalities that:

$$
\left|\frac{a_{n+1}}{a_{n+2}}\right|-\left|\frac{a_{n}}{a_{n+1}}\right| \geq\left(\frac{1}{|\alpha|}\right)^{E\left(\left(\frac{n+2}{N}\right)^{N}-\left(\frac{n+1}{N}\right)^{N}\right)}-\left(\frac{1}{|\alpha|}\right)^{E\left(\left(\frac{n+1}{N}\right)^{N}-\left(\frac{n}{N}\right)^{N}\right)+1}
$$


But, by Lemma 2.10., We have:

$$
\begin{aligned}
& E\left(\left(\frac{n+2}{N}\right)^{N}-\left(\frac{n+1}{N}\right)^{N}\right)-E\left(\left(\frac{n+1}{N}\right)^{N}-\left(\frac{n}{N}\right)^{N}\right)-1 \geq \\
& E\left(\left[\left(\frac{n+2}{N}\right)^{N}-\left(\frac{n+1}{N}\right)^{N}\right]-\left[\left(\frac{n+1}{N}\right)^{N}-\left(\frac{n}{N}\right)^{N}\right]\right)-1 . \\
& \text { As : }\left(\frac{n+2}{N}\right)^{N}-\left(\frac{n+1}{N}\right)^{N}=\frac{1}{N^{N}} \sum_{i=0}^{N-1}(n+2)^{i}(n+1)^{N-i-1} \text { and } \\
& \left(\frac{n+1}{N}\right)^{N}-\left(\frac{n}{N}\right)^{N}=\frac{1}{N^{N}} \sum_{i=0}^{N-1} n^{i}(n+1)^{N-i-1}, \text { we see that: } \\
& {\left[\left(\frac{n+2}{N}\right)^{N}-\left(\frac{n+1}{N}\right)^{N}\right]-\left[\left(\frac{n+1}{N}\right)^{N}-\left(\frac{n}{N}\right)^{N}\right]=\frac{1}{N^{N}} \sum_{i=0}^{N-1}(n+1)^{N-i-1}\left[(n+2)^{i}-n^{i}\right] .}
\end{aligned}
$$

Hence:

$$
\begin{aligned}
{\left[\left(\frac{n+2}{N}\right)^{N}-\left(\frac{n+1}{N}\right)^{N}\right]-\left[\left(\frac{n+1}{N}\right)^{N}-\left(\frac{n}{N}\right)^{N}\right]=} & \\
& \frac{2}{N^{N}} \sum_{i=0}^{N-1}(n+1)^{N-i-1} \sum_{j=0}^{i-1}(n+2)^{j} n^{i-j-1} .
\end{aligned}
$$

It follows that:

$$
\begin{aligned}
{\left[\left(\frac{n+2}{N}\right)^{N}-\left(\frac{n+1}{N}\right)^{N}\right]-\left[\left(\frac{n+1}{N}\right)^{N}-\left(\frac{n}{N}\right)^{N}\right] \geq } & \\
& \frac{2}{N^{N}} \sum_{i=0}^{N-1} n^{N-i-1} \sum_{j=0}^{i-1} n^{j} n^{i-j-1},
\end{aligned}
$$

then that:

$$
\left[\left(\frac{n+2}{N}\right)^{N}-\left(\frac{n+1}{N}\right)^{N}\right]-\left[\left(\frac{n+1}{N}\right)^{N}-\left(\frac{n}{N}\right)^{N}\right] \geq \frac{2 n^{N-2}}{N^{N}} \sum_{i=0}^{N-1} i,
$$

and finally:

$$
\left[\left(\frac{n+2}{N}\right)^{N}-\left(\frac{n+1}{N}\right)^{N}\right]-\left[\left(\frac{n+1}{N}\right)^{N}-\left(\frac{n}{N}\right)^{N}\right] \geq \frac{n^{N-2}(N-1)}{N^{N-1}} .
$$

It follows that for every $n \geq n_{0}$, we have:

$$
\left[\left(\frac{n+2}{N}\right)^{N}-\left(\frac{n+1}{N}\right)^{N}\right]-\left[\left(\frac{n+1}{N}\right)^{N}-\left(\frac{n}{N}\right)^{N}\right] \geq 2 .
$$

Consequently for every $n \geq n_{0}$, we have:

$$
E\left(\left(\frac{n+2}{N}\right)^{N}-\left(\frac{n+1}{N}\right)^{N}\right)-E\left(\left(\frac{n+1}{N}\right)^{N}-\left(\frac{n}{N}\right)^{N}\right)-1>0 .
$$

It follows that for every $n \geq n_{0}$, we have: $\left|\frac{a_{n+1}}{a_{n+2}}\right|>\left|\frac{a_{n}}{a_{n+1}}\right|$.

We complete the proof by applying Theorem 2.9.

In what follows, one aims to show that, given a $p$-adic transcendental entire function $f$, it is always easy to transform it into a prime entire function. For this, most often, we have just to add, or multiply $f$ by an affinity. 
Theorem 2.15. Let $f$ be a p-adic transcendental entire function. Then the set $\left\{a \in \mathbb{C}_{p} ; f(x)-a x\right.$ is not prime $\}$ is at most a countable set.

To prove Theorem 2.15 we need the following lemma.

Lemma 2.16. Let $f \in \mathcal{A}\left(\mathbb{C}_{p}\right) \backslash \mathbb{C}_{p}[X]$. Then there exists a countable set $E \subset \mathbb{C}_{p}$ such that for every $a \in \mathbb{C}_{p} \backslash E$ and every $b \in \mathbb{C}_{p}$, the function $f(x)-(a x+b)$ has at most one multiple zero.

Proof. Let $Z\left(f^{\prime \prime}\right)$ be the set of zeros of $f^{\prime \prime}$. Since $\mathbb{C}_{p}$ is a separable space, there exists a countable family of disks $\left(d_{i}\right)_{i \geq 1}$ such that $\mathbb{C}_{p} \backslash Z\left(f^{\prime \prime}\right)=\cup_{i \geq 1} d_{i}$ and, for every $i \geq 1$, the restriction $f_{i}^{\prime}$ of $f^{\prime}$ to $d_{i}$ is a bi-analytic function on $d_{i}$. Then we have $\mathbb{C}_{p}=\left(\cup_{i \geq 1} D_{i}\right) \cup f^{\prime}\left(Z\left(f^{\prime \prime}\right)\right)$.

Let $g$ be the function defined on $\mathbb{C}_{p}$ by $g(x)=f(x)-x f^{\prime}(x)$. It should be noted that even if the family $\left(d_{i}\right)_{i \geq 1}$ is chosen so that the disks $d_{i}$ are pairwise disjoint, there is no guarantee that the family $\left(D_{i}\right)_{i \geq 1}$ retains this property. In other words some of the disks $D_{i}$ could be nested. To take account of this fact, let us set: $\Gamma=\left\{(i, j) \in\left(\mathbb{N}^{*}\right)^{2} / D_{i} \varsubsetneqq D_{j}\right.$ and $g \circ\left(f_{i}^{\prime}\right)^{-1} \neq g \circ\left(f_{j}^{\prime}\right)^{-1}$ in $\left.D_{i}\right\} ;$
$\Delta_{i j}=\left\{x \in D_{i} / g \circ\left(f_{i}^{\prime}\right)^{-1}(x)=g \circ\left(f_{j}^{\prime}\right)^{-1}(x)\right\}, \forall(i, j) \in \Gamma$ and $\Delta=\cup_{(i, j) \in \Gamma} \Delta_{i j}$. Then $E=\mathbb{C}_{p} \backslash\left[\left(\cup_{i \geq 1} D_{i}\right) \backslash\left[\left(f^{\prime}\left(Z\left(f^{\prime \prime}\right)\right)\right) \cup \Delta\right]\right]$ is a countable subset of $\mathbb{C}_{p}$. Indeed it is easily seen that $E \subset\left(f^{\prime}\left(Z\left(f^{\prime \prime}\right)\right)\right) \cup \Delta$.

Let $a \in \mathbb{C}_{p} \backslash E$. Let $I_{a}=\left\{i \in \mathbb{N}^{*} / a \in D_{i}\right\}$. Hence the disks $D_{i}$, for $i \in I_{a}$, are nested. We easily show that the set of multiple zeros of the function $f(x)-(a x+b)$ is equal to $A=\left\{\left(f_{i}^{\prime}\right)^{-1}(a) / i \in I_{a}\right.$ and $\left.f \circ\left(f_{i}^{\prime}\right)^{-1}(a)=a\left(f_{i}^{\prime}\right)^{-1}(a)+b\right\}$.

Suppose that $\left(f_{i}^{\prime}\right)^{-1}(a)$ and $\left(f_{j}^{\prime}\right)^{-1}(a)$ are two distinct elements of $A$. We may assume that $D_{i} \varsubsetneqq D_{j}$. The fact that each of these elements is a solution of the equation $f(x)-a x=b$ implies that: $f\left(\left(f_{i}^{\prime}\right)^{-1}(a)\right)-\left(f_{i}^{\prime}\right)^{-1}(a) f^{\prime}\left(\left(f_{i}^{\prime}\right)^{-1}(a)\right)=$ $f\left(\left(f_{j}^{\prime}\right)^{-1}(a)\right)-\left(f_{j}^{\prime}\right)^{-1}(a) f^{\prime}\left(\left(f_{j}^{\prime}\right)^{-1}(a)\right)$ or, in other words, $g \circ\left(f_{i}^{\prime}\right)^{-1}(a)=g \circ$ $\left(f_{j}^{\prime}\right)^{-1}(a)$. As $a \notin \Delta$, we deduce that $g \circ\left(f_{i}^{\prime}\right)^{-1}(x)=g \circ\left(f_{j}^{\prime}\right)^{-1}(x), \forall x \in D_{i}$.

By derivation of this last equation, we have: $\left(f_{i}^{\prime}\right)^{-1}(x)=\left(f_{j}^{\prime}\right)^{-1}(x), \forall x \in D_{i}$ and particularly $\left(f_{i}^{\prime}\right)^{-1}(a)=\left(f_{j}^{\prime}\right)^{-1}(a)$, which is a contradiction. Hence the set $A$ admits at most one element. This completes the proof Lemma 2.16. 
Proof of Theorem 2.15

Let $E$ be the countable set of Lemme 2.16. and let $a \in \mathbb{C}_{p} \backslash E$. Since $f$ is a transcendental entire function, we see that the function $h(x)=f(x)-a x$ is also a transcendental entire function. Lemma 2.16 then ensures that, for every $b \in \mathbb{C}_{p}$, the function $h(x)-b=f(x)-a x-b$ admits at most one multiple zero. Theorem 2.6 finally enables us to conclude that the function $f(x)-a x=h(x)$ is prime.

Theorem 2.17. Let $f$ be a p-adic transcendental entire function. Then the set $\left\{a \in \mathbb{C}_{p} ; f(x)(x-a)\right.$ is not prime $\}$ is at most a countable set.

To prove Theorem 2.17 we need the following lemma.

Lemma 2.18. Let $f \in \mathcal{A}\left(\mathbb{C}_{p}\right) \backslash \mathbb{C}_{p}[X]$. Then there exists a countable set $E^{\prime} \subset \mathbb{C}_{p}$ such that for every $a \in \mathbb{C}_{p} \backslash E^{\prime}$ and every $b \in \mathbb{C}_{p}$, the function $(x-a) f(x)-b$ has at most one multiple zero.

Proof. The procedure is very similar to that used in the proof of Lemma 2.16. We easily check that an element $\zeta$ of $\mathbb{C}_{p}$ is a multiple zero of the function $(x-a) f(x)-b$ if and only if $\left\{\begin{array}{l}a=g(\zeta) \\ b=h(\zeta)\end{array}\right.$, where $g$ and $h$ are meromorphic functions, defined in $\mathbb{C}_{p}$, by:

$\left\{\begin{array}{l}g(x)=x+f(x) / f^{\prime}(x) \\ h(x)=(x-g(x)) f(x)\end{array}\right.$

Let $S\left(g^{\prime}\right)$ be the set of zeros and poles of $g^{\prime}$ and let $\left(d_{i}\right)_{i \geq 1}$ be a countable family of disks such that $\mathbb{C}_{p} \backslash S\left(g^{\prime}\right)=\cup_{i \geq 1} d_{i}$ and that, for every $i \geq 1$, the restriction $g_{i}$ of $g$ to $d_{i}$ is a bi-analytic function on $d_{i}$. Then we have: $\mathbb{C}_{p}=$ $\left(\cup_{i \geq 1} D_{i}\right) \cup g\left(S\left(g^{\prime}\right)\right)$, where $D_{i}=g\left(d_{i}\right)$.

As noted before, the disks $D_{i}$ are not necessarily pairwise disjoint. In other words, some of them could be nested. To take account of this fact, let us set:

$$
\begin{gathered}
\Gamma=\left\{(i, j) \in\left(\mathbb{N}^{*}\right)^{2} / D_{i} \varsubsetneqq D_{j} \text { and } h \circ g_{i}^{-1} \neq h \circ g_{j}^{-1} \text { in } D_{i}\right\} ; \\
\Delta_{i j}=\left\{x \in D_{i} / h \circ g_{i}^{-1}(x)=h \circ g_{j}^{-1}(x)\right\}, \forall(i, j) \in \Gamma \text { and } \Delta=\cup_{(i, j) \in \Gamma} \Delta_{i j} .
\end{gathered}
$$

Then $E^{\prime}=\mathbb{C}_{p} \backslash\left[\left(\cup_{i \geq 1} D_{i}\right) \backslash\left[\left(g\left(S\left(g^{\prime}\right)\right)\right) \cup \Delta \cup\left(\cup_{i \geq 1} Z\left(f \circ g_{i}^{-1}\right)\right)\right]\right]$ is a countable subset of $\mathbb{C}_{p}$. Indeed, it is clear that $\left.E^{\prime} \subset\left(g\left(S\left(g^{\prime}\right)\right)\right) \cup \Delta \cup\left(\cup_{i \geq 1} Z\left(f \circ g_{i}^{-1}\right)\right)\right]$. 
Let $a \in \mathbb{C}_{p} \backslash E^{\prime}$. Let $I_{a}=\left\{i \in \mathbb{N}^{*} / a \in D_{i}\right\}$. Hence the disks $D_{i}$, for $i \in I_{a}$, are nested. We easily show, by relation (1), that the set of multiple zeros of the function $(x-a) f(x)-b$ is equal to $A=\left\{g_{i}^{-1}(a) / b=h \circ g_{i}^{-1}(a)\right.$ for $\left.i \in I_{a}\right\}$.

Suppose that $g_{i}^{-1}(a)$ and $g_{j}^{-1}(a)$ are two distinct elements of $A$. Hence, we have: $h\left(g_{i}^{-1}(a)\right)=h\left(g_{j}^{-1}(a)\right)=b$. Assuming that $D_{i} \varsubsetneqq D_{j}$ and using the fact that $a \notin \Delta$, we have:

$h \circ g_{i}^{-1}(x)=h \circ g_{j}^{-1}(x), \forall x \in D_{i}$

From relation (1), we have:

$h^{\prime}=-f g^{\prime}$

By derivation of relation (2) and using relation (3), we obtain:

$f \circ g_{i}^{-1}(x)=f \circ g_{j}^{-1}(x), \forall x \in D_{i}$

Particularly, we have $f \circ g_{i}^{-1}(a)=f \circ g_{j}^{-1}(a)$. But, since $a \in E^{\prime}$, we have $f \circ g_{i}^{-1}(a)=f \circ g_{j}^{-1}(a) \neq 0$. Using this, we deduce from relation (1) that: $g_{i}^{-1}(a)=g_{j}^{-1}(a)$, a contradiction. Hence the set $A$ admits at most one element. This completes the proof Lemma 2.18 .

Proof of Theorem 2.17

Let $E$ be the countable set of Lemme 2.18 and let $a \in \mathbb{C}_{p} \backslash E^{\prime}$. Since $f$ is a transcendental entire function, we see that the function $h(x)=f(x)(x-a)$ is also a transcendental entire function. Lemma 2.18 then ensures that, for every $b \in \mathbb{C}_{p}$, the function $h(x)-b=f(x)(x-a)-b$ admits at most one multiple zero. Theorem 2.6 finally enables us to conclude that the function $f(x)(x-a)=h(x)$ is prime.

\section{PERMUTABILITY OF ENTIRE FUNCTIONS}

In this part, we consider the question of permutability of $p$-adic meromorphic functions. In other words, given two $p$-adic meromorphic functions $f$ and $g$, under what conditions can we have $f \circ g=g \circ f$ ? The very particular situation that we are going to study gives an idea of the extreme difficulty of exploring this problem in a general way. More precisely, we will show the following result:

Theorem 3.1. $\quad$ Let $P$ and $f$ be respectively a non-constant polynomial and $a$ transcendental entire function on $\mathbb{C}_{p}$ such that $P \circ f=f \circ P$. Then the polynomial $P$ is of one of the following forms: 
i) $P(x)=x$,

ii) $P(x)=a x+b$, where $a, b \in \mathbb{C}_{p}$ such that $a$ is an $n$-th root of unity for some non-zero integer $n$.

To prove this theorem, we neen the following lemmas:

Lemma 3.2. Let $f \in \mathcal{A}\left(\mathbb{C}_{p}\right)$. Then the two following assertions are equivalent:

i) $f$ is a polynomial,

ii) there exist $c>0$ and $d \geq 0$ such that $|f|(r) \leq c r^{d}, r \rightarrow+\infty$.

Proof. Suppose that $f$ is a polynomial of degree $n: f(x)=a_{0}+\ldots+a_{n} x^{n}, a_{n} \neq 0$. Since, for $r$ large enough, we have $|f|(r)=\left|a_{n}\right| r^{n}$, just take $c=\left|a_{n}\right|$ and $d=n$ to have the desired inequality.

Conversely, if there are $c>0$ and $d \geq 0$ such that $|f|(r) \leq c r^{d}$ as $r \rightarrow+\infty$, we have for every integer $n>d$ : $\left|f^{(n)}\right|(r) \leq|f|(r) / r^{n} \leq c r^{d-n} \longrightarrow 0$. So $f^{(n)} \equiv 0$ and $f$ is a polynomial.

Lemma 3.3. Let $f \in \mathcal{A}\left(\mathbb{C}_{p}\right)$ and suppose that there exist real numbers $\alpha, \beta>0$ and an integer $n \geq 2$ such that, $|f|\left(\alpha r^{n}\right)<\beta(|f|(r))^{n}$, when $r \rightarrow+\infty$. Then $f$ is a polynomial.

Proof. We will construct real numbers $c, d>0$ such that $|f|(r) \leq c r^{d}, r \rightarrow+\infty$. Let $r_{0}$ be a real number such that $r_{0}>\max \left\{1, \alpha^{1 /(1-n)}\right\}$. Let us choose $c, d>0$ such that $\left\{\begin{array}{l}|f|\left(r_{0}\right)<c r_{0}^{d} \\ \beta<\alpha^{d} / c^{n-1}\end{array}\right.$, and let us set $r_{k+1}=\alpha r_{k}^{n}$ for $k \geq 0$.

The sequence $\left(r_{k}\right)$ est strictly increasing and tends to $+\infty$. We prove by induction that: $|f|\left(r_{k}\right)<c r_{k}^{d}, \forall k \geq 0$. Indeed, the property holds for $k=0$. Suppose that it holds for some integer $k \geq 0$. Then we have:

$$
\begin{aligned}
|f|\left(r_{k+1}\right)= & |f|\left(\alpha r_{k}^{n}\right)<\beta\left(|f|\left(r_{k}\right)\right)^{n}<\beta c^{n} r_{k}^{d n}=c\left(\beta c^{n-1}\right) r_{k}^{d n} \\
& <c \alpha^{d} r_{k}^{d n}=c\left(\alpha r_{k}^{n}\right)^{d}=c r_{k+1}^{d},
\end{aligned}
$$

which means that this inequality is true for $k+1$.

For every $r \in\left[r_{k}, r_{k+1}\right]$, there exists $t \in[0 ; 1]$ such that $r=r_{k}^{t} r_{k+1}^{1-t}$. As $\log r \mapsto \log |f|(r)$ is a convex function, we deduce that: 


$$
\begin{aligned}
|f|(r) & \leq\left(|f|\left(r_{k}\right)\right)^{t}\left(|f|\left(r_{k+1}\right)\right)^{1-t}=\left(|f|\left(r_{k}\right)\right)^{t}\left(|f|\left(\alpha r_{k}^{n}\right)\right)^{1-t} \\
& <\left(|f|\left(r_{k}\right)\right)^{t}\left(\beta\left(|f|\left(r_{k}\right)\right)^{n}\right)^{1-t}<\left(c r_{k}^{d}\right)^{t}\left(\beta\left(c r_{k}^{d}\right)^{n}\right)^{1-t} \\
& =c^{t} r_{k}^{d t} c^{1-t}\left(\beta c^{n-1}\right)^{1-t}\left(r_{k}^{n(1-t)}\right)^{d}<c r_{k}^{d t} \alpha^{d(1-t)} r_{k}^{n(1-t) d} \\
& =c\left(r_{k}^{t}\left(\alpha r_{k}^{n}\right)^{1-t}\right)^{d}=c\left(r_{k}^{t} r_{k+1}^{1-t}\right)^{d}=c r^{d} .
\end{aligned}
$$

Hence $|f|(r)<c r^{d}, \forall r \geq r_{0}$. By Lemma 3.2, $f$ is then a polynomial.

\section{Proof of Theorem 3.1.}

Let us set $P(x)=a_{0}+\cdots+a_{k} x^{k}$, where $a_{0}, \ldots, a_{k}$ are elements of $\mathbb{C}_{p}$ and $a_{k} \neq 0$. Then: $(f \circ P)(x)=(P \circ f)(x)=a_{0}+\cdots+a_{k}(f(x))^{k}$. Suppose that $k \geq 2$. Then, for $r>0$, we have $|f|(|P|(r))=|P|(|f|(r))$. It follows that, for sufficiently large $r>0$, we have $|f|\left(\left|a_{k}\right| r^{k}\right)=\left|a_{k}\right|(|f|(r))^{k}<2\left|a_{k}\right|(|f|(r))^{k}$. Lemma 3.3 then implies that $f$ is a polynomial, , which is a contradiction. Hence, we have $k=1$ and $P$ is of the form $P(x)=a x+b$, where $a, b \in \mathbb{C}_{p}$ and $a \neq 0$. Two cases can then arise:

i) $a=1$. Then we have $b=0$. Indeed suppose that $b \neq 0$. Then we have, for every $x \in \mathbb{C}_{p}, f(x+b)=f(x)+b$. It follows that $f^{\prime}(x+b)=f^{\prime}(x)$, for every $x \in \mathbb{C}_{p}$. Let $\zeta$ be a zero of $f^{\prime}$ such that $|\zeta|>|b|$. Then $\zeta, \zeta+b, \zeta+2 b, \ldots$ are infinitely many zeros of $f^{\prime}$ included in the disk $d(0 ;|\zeta|)$, a contradiction. Hence in this case we have $P(x)=x$.

ii) $a \neq 1$. Let $\sigma$ be the affine application $\sigma(t)=t+b(a-1)^{-1}$, hence its inverse $\sigma^{-1}$ is given by $\sigma^{-1}(t)=t+b(1-a)^{-1}$. Let $F$ be the function given by $F=\sigma \circ f \circ \sigma^{-1}$. It is easily seen that: $F(a x)=a F(x), \forall x \in \mathbb{C}_{p}$.

If $F(x)=\sum_{n \geq 0} b_{n} x^{n}$, we have $F(a x)=\sum_{n \geq 0} b_{n} a^{n} x^{n}$. It follows that:

$\sum_{n \geq 0}\left(a^{n}-a\right) b_{n} x^{n}=0$, and hence $b_{0}=0$ and $\left(a^{n}-a\right) b_{n+1}=0, \forall n \geq 1$.

Suppose that there exist two relatively prime integers $m, n \geq 2$ such that $b_{n+1} \neq 0$, and $b_{m+1} \neq 0$ we would have $a^{n}=a^{m}=1$, and therefore $a=1$, which excluded. Hence $F$ has the form $F(X)=\sum_{k \geq 1} b_{n k+1} x^{n k+1}$, where $n$ is the smallest positive integer such that $a^{n}=1$. It follows that:

$$
f(x)=\sum_{k \geq 1} b_{n k+1}(x+b /(a-1))^{n k+1}+b /(1-a) .
$$


Corollary 3.4. Let $f \in \mathcal{A}\left(\mathbb{C}_{p}\right) \backslash \mathbb{C}_{p}[X], f(x)=\sum_{n \geq 0} a_{n} x^{n}$. Suppose that there exist two relatively prime integers $m, l$ such that $a_{m} a_{l} \neq 0$. Then the only non-constant polynomial $P$ such that $P \circ f=f \circ P$ is $P(x)=x$.

\section{References}

[1] Y. Noda, On the factorization of entire functions. Kodai Math. J. 4. (1981), 480-494

[2] J. P. Bézivin and A. Boutabaa, Decomposition of p-adic meromorphic functions, Ann. Math. Blaise Pascal, vol.2, N1, 1995, pp. 51-60

[3] J. P. Bézivin, Wronskien et équations différentielles p-adiques, Acta Arithmetica 158 no 1 (2013), pp.61-pp.78.

[4] A. Escassut. Analytic Elements in p-adic Analysis. World Scientific Publishing Co. Pte. Ltd. (Singapore, 1995).

[5] A. Escassut and J. Ojeda. Exceptional values of p-adic analytic functions and derivatives.Complex variables and eleptic functions, 56, no 1-4 (2011), 263269.

[6] M. Ozawa. On certain criteria for the left-primeness of entire functions. Kodai Math. Sem. Rep, 26 (1975), 304-317

[7] M. Ozawa. On certain criteria for the left-primeness of entire functions. II. Kodai Math. Sem. Rep, 27 (1976), 1-10

Bilal SAOUDI

Laboratoire de Mathématiques Pures et Appliquées,

Université de Jijel, BP 98, Jijel, Algérie.

Email:saoudibilal1@gmail.com

Abdelbaki BOUTABAA 
Université Clermont Auvergne LMBP UMR 6620 - CNRS

Campus universitaire des Czeaux

3, Place Vasarely

TSA 60026

CS 60026

63178, Aubire-Cedex; France

Email: Abdelbaki.Boutabaa@math.univ-bpclermont.fr

Tahar Zerzaihi

Laboratoire de Mathématiques Pures et Appliquées,

Université de Jijel,

BP 98, Jijel, Algérie.

Email:zerzaihi@yahoo.com 